\title{
Advanced Electronic Interconnection
}

\author{
SHIH-KANG LIN (i] $1,2,3,4$ \\ 1.-Department of Materials Science and Engineering, National Cheng Kung University, Tainan \\ City 70101, Taiwan. 2.-Center for Micro/Nano Science and Technology, National Cheng Kung \\ University, Tainan City 70101, Taiwan. 3.-Hierarchical Green-Energy Materials (Hi-GEM) \\ Research Center, National Cheng Kung University, Tainan City 70101, Taiwan. 4.-e-mail: \\ linsk@mail.ncku.edu.tw
}

Advanced electronic interconnection has been the cutting-edge technology in electronic packaging for a number of new applications, ranging from $5 \mathrm{G}$ communications, artificial intelligence, internet-ofthings, supercomputing, and data centers to power control units for renewable energies and electric vehicles. These new applications have brought great challenges on the materials associated with the new interconnection schemes. For example, three-dimensional integrated circuits technology and fan-out wafer-level packaging technology have been proposed for high-density chips in promoting Moore's law to the "more-than-Moore" era, which would involve the direct bonding process, particularly $\mathrm{Cu}$ to-Cu bonding, instead of conventional soldering. On the other hand, die-attachment processes for wide band-gap (WBG) semiconductors in power modulus involve sintering bonding for metallic pastes. Both developments in advanced electronic interconnection have drastically increased the current density exerted on the materials as well as the local operating temperatures for electronic interconnections.

In the present topic, Advanced Electronic Interconnection, a well-rounded combination of invited and unsolicited papers, including one review article, has been compiled. It aims to provide JOM readers with a collection of recent developments of the aforementioned emerging materials and technologies for advanced electronic interconnection.

Mechanical properties of electronic joints are crucial for the reliability of electronic products. In particular, Sn-based solders based on a body-centered tetragonal structure as the matrix with high anisotropic properties are used in various levels of electronic interconnections. The first invited article, "Creep Behaviors Along Characteristic Crystal

Shih-kang Lin is the JOM advisor for the Alloy Phases Committee, a joint committee of the TMS Function Materials Division and the Structural Materials Division, and guest editor for the topic Advanced Electronic Interconnection in this issue.
Orientations of Sn and Sn-1.8Ag by Using Nanoindentation" by P.J. Chiang et al., investigates the creep properties of pure Sn and the Sn-1.8Ag alloy along (100) and (001) orientations using nanoindentation. The slip systems were also revealed using transmission electron microscopy.

Interfaces in electronic joints, such as the solder/substrate contacts, play an important role in the strength, toughness, electronic conductivity, thermal conductivity, etc. of the electronic interconnections. The interfacial reactions between multicomponent solders and alloys or multi-layer substrates bring significant complexity of interfacial phase formation, microstructural evolution, and defect formation. The second article, "Suppression of Void Formation at Sn/ $\mathrm{Cu}$ Joint Due to Twin Formation in $\mathrm{Cu}$ Electrodeposit" by S.-T. Tsai et al., investigates the interfacial reactions between $\mathrm{Sn}$ and $\mathrm{Cu}$ electroplated substrates based on various additive formulae. $\mathrm{A} \mathrm{Cu}$ substrate with a high density of twins was fabricated, and the formation of voids in the subsequent aging test was suppressed. The third article, "Effects of Minor $\mathrm{Cu}, \mathrm{Ni}$ and $\mathrm{Ag}$ Additions on the Reactions between Sn-Based Solders and Co Substrate" by C.-h. Wang et al., reports on the alloying effect of minor $\mathrm{Cu}$, $\mathrm{Ni}$, and $\mathrm{Ag}$ on pure $\mathrm{Sn}$ in liquid/solid and solid/solid reactions with Co substrates. The reaction phase formation and intermetallic compound (IMC) growth kinetics in these reaction couples are presented. The fourth article, "Interfacial Reactions Between LeadFree Solders and Ni-Pd-Co Alloys" by Y.-W. Yen et al., studies the interfacial reactions between three types of Pb-free solders, namely $\mathrm{Sn}, \mathrm{Sn}-3 \mathrm{Ag}-0.5 \mathrm{Cu}$ and $\mathrm{Sn}-$ $9 \mathrm{Zn}$, and Ni- $x \mathrm{Pd}-y$ Co substrates, where $x=0.5$ and 1.5 , and $y=0.5,1.5,3.0$ wt. $\%$ at $240^{\circ} \mathrm{C}, 270^{\circ} \mathrm{C}$, and $300^{\circ} \mathrm{C}$. The reaction phase formation at the interfaces of the couples were identified and the corresponding kinetics of IMC growth reported.

The formation of Sn whiskers is one of the causes of failure of electronic devices. It can be induced by various sources of compressive stress, which have 
been extensively investigated for decades. The fifth article, "Effect of Sn Film Grain Size and Thickness on Kinetics of Spontaneous Sn Whisker Growth" by W.C. Lin et al., examines the effects of the grain size and thickness of a Sn thin film on the growth of Sn whiskers. The Sn thin films were electroplated on pure $\mathrm{Cu}$ and the whiskers were formed at the artificially made opening after being annealed at $40^{\circ} \mathrm{C}$. An equation is proposed to evaluate the effects of grain size and thickness on the Sn whisker growth.

Die attachment technology has been a hot topic in WBG semiconductor packaging and application. The conventional soldering process cannot fulfill the requirements in high-power and high-temperature applications. Sintering bonding using Ag-based particles or pastes has been employed to resolve the limitation of conventional soldering. One approach is to produce a high-temperature joint via the formation of a high-temperature phase through reactions. The sixth article,"Rapid Formation of Full Intermetallic Bondlines for Die Attachment in High-Temperature Power Devices Based on Microsized Sn-Coated Ag Particles" by F. Yu et al., proposes a bonding process using Sn-coated $\mathrm{Ag}$ particles to form a full $\mathrm{Ag}_{3} \mathrm{Sn} \mathrm{IMC}$ joint. The quality of the sintering is crucial for the joint properties. The seventh article, "Interconnect Fabrication on Polymer Substrate using Submicron/Nano Silver Particles with the Assistance of Low-Density Irradiations" by G.-L. Huang et al., investigates the effect of irradiation on the sintering bonding with submicron/nano-Ag particles for polymeric substrates. The reliability of the joints of die attachment, particularly after thermal cycling or power cycling, needs to be assessed. The eighth article, "Thermal Cycling of Sintered Silver (Ag) Joint as Die-Attach Material" by K.S. Siow and S.T. Chua, investigates the sintered $\mathrm{Ag}$ joints after thermal cycling. While Ag particles have mostly been studied for die attachment, the exploration of employing cheaper $\mathrm{Cu}$ particles never ceases. The ninth article, "Facile Preparation of Self-Reducible Cu Nanoparticle Paste for Low Temperature $\mathrm{Cu}-\mathrm{Cu}$ Bonding" by Y. Mou et al., proposes an approach for low-temperature bonding with $\mathrm{Cu}$.

Electronic products and electronic joints, by definition, experience electric currents during the operation of the devices. The electromigration effect induced by electric currents is one of the major failure modes, especially in high-density and highpower electronic interconnections. The tenth article, "Electromigration Behavior of Screen-Printing Silver Nanoparticles Interconnects" by W.-H. Lin and F.-Y. Ouyang, investigates the electromigration effect upon the conduction lines fabricated by screen-printing Ag particles. The last article, "A Critical Review on the Electromigration Effect, the Electroplastic Effect, and Perspectives on the Effects of Electric Current upon Alloy Phase Stability" by Y.-c. Liu and S.-k. Lin, undertakes a critical review on the recent development on the electric current effects upon material phase stability, and also provides perspectives on the topic.

The following papers being published under the topic of Advanced Electronic Interconnection provide excellent details and research on the subject. To download any of the papers, follow the URL http://ink.springer.com/journal/11837/71/9/page/1 to the table of contents page for the September 2019 issue (vol. 71, no. 9).

- "Creep Behaviors Along Characteristic Crystal Orientations of $\mathrm{Sn}$ and $\mathrm{Sn}-1.8 \mathrm{Ag}$ by Using Nanoindentation" by P.J. Chiang, J.Y. Wu, H.Y. Yu, and C.R. Kao.

- "Suppression of Void Formation at $\mathrm{Sn} / \mathrm{Cu}$ Joint Due to Twin Formation in Cu Electrodeposit" by Shan-Ting Tsai, Ping-Chen Chiang, Chang Liu, Shien-Ping Feng, and Chih-Ming Chen.

- "Effects of Minor $\mathrm{Cu}, \mathrm{Ni}$ and $\mathrm{Ag}$ Additions on the Reactions between Sn-Based Solders and Co Substrate" by Chao-hong Wang, Chun-yi Kuo, and Yu-bin Guo.

- "Interfacial Reactions Between Lead-Free Solders and Ni-Pd-Co Alloys" by Yee-Wen Yen, Hsien-Ming Hsiao, Kuo-Jung Chen, Yi-Show Lin, and Mei-Ting Lai.

- "Effect of Sn Film Grain Size and Thickness on Kinetics of Spontaneous Sn Whisker Growth" by Wen Chih Lin, Tsan-Hsien Tseng, Wei Liu, KuoShuo Huang, Hao Chen, Hsin Yi Lee, Ching Shun $\mathrm{Ku}$, and Albert T. Wu.

- "Rapid Formation of Full Intermetallic Bondlines for Die Attachment in High-Temperature Power Devices Based on Micro-sized Sn-Coated Ag Particles" by Fuwen Yu, Hao Liu, Chunjin Hang, Hongtao Chen, and Mingyu Li.

- "Interconnect Fabrication on Polymer Substrate using Submicron/Nano Silver Particles with the Assistance of Low-Density Irradiations" by GuoLun Huang, Po-Hsiang Chiu, Masahisa Fujino, and Jenn-Ming Song.

- "Thermal Cycling of Sintered Silver (Ag) Joint as Die-Attach Material" by K.S. Siow and S.T. Chua.

- "Facile Preparation of Self-Reducible $\mathrm{Cu}$ Nanoparticle Paste for Low Temperature $\mathrm{Cu}$ $\mathrm{Cu}$ Bonding" by Yun Mou, Jiaxin Liu, Hao Cheng, Yang Peng, and Mingxiang Chen.

- "Electromigration Behavior of Screen-Printing Silver Nanoparticles Interconnects" by WanHsuan Lin and Fan-Yi Ouyang.

- "A Critical Review on the Electromigration Effect, the Electroplastic Effect, and Perspectives on the Effects of Electric Current upon Alloy Phase Stability" by Yu-chen Liu and ShihKang Lin.

Publisher's Note Springer Nature remains neutral with regard to jurisdictional claims in published maps and institutional affiliations. 